Rev. Saúde públ., S. Paulo, 19:304-10, 1985.

\title{
TEMPO DE ALCOOLISMO NO DESENVOLVIMENTO DE DOENÇAS ORGÂNICAS EM MULHERES TRATADAS NO HOSPITAL DAS CLINICAS DE RIBEIRĀO PRETO, SP, BRASIL
}

Roberto Oliveira Dantas*

\begin{abstract}
DANTAS, R.O. Tempo de alcoolismo no desenvolvimento de doenças orgânicas em mulheres tratadas no Hospital das Clínicas de Ribeirão Preto, SP, Brasil. Rev. Saúde públ., S. Paulo, $19: 304-10,1985$.
\end{abstract}

RESUMO: Com o objetivo de conhecer o tempo de ingestão freqüente de bebidas alcoólicas (ingestão média de mais de $100 \mathrm{ml}$ de etanol por dia, pelo menos três dias por semana), até o aparecimento de sinais e sintomas de doenças orgânicas consequientes ao hábito, estudamos 95 mulheres tratadas entre 1978 e 1982 no Hospital das Clínicas de Ribeirão Preto, portadoras de doenças orgânicas associadas ao alcoolismo. Foi feito diagnóstico clínico e laboratorial de cirosse hepática em 32 pacientes, de pancreatite crônica em 13 e de outras doenças (pelagra, desnutrição, neurite periférica e hepatite alcoólica) em 50. Pacientes com apenas sintomas psiquiátricos não foram estudadas. A obtenção das informações ocorreu após alguns dias de tratamento. Em média a idade em que começaram a ter sinais e sintomas das doenças que motivaram a procura de hospital para tratamento foi de $35,30 \pm 7,72$ anos na pancreatite crônica, $36,53 \pm 8,39$ anos na cirrose hepática e de $33,90 \pm 11,27$ anos nas outras doenças. O tempo de ingestão da bebida foi de 15,92 $\pm 7,15$ anos na pancreatite crônica, 14,62 $\pm 8,70$ anos na cirrose hepática e 13,24 $\pm 9,58$ anos nas outras doenças. Antecedentes familiares de alcoolismo estiveram presentes em $64,2 \%$ dos casos, geralmente marido ou companheiro. Nenhuma delas tinha outras mulheres na família com problemas de alcoolismo. A média do tempo de alcoolismo para o aparecimento de cirrose hepática nas mulheres (14,62 anos) foi menor do que a encontrada para homens da mesma população $(21,10$ anos), estudados em trabalhos anteriores.

UNITERMOS: Alcoolismo. Mulheres. Ảlcool, ingestão. Cirrose hepática alcoólica. Pancreatite crônica.

\section{INTRODUÇÃO}

O alcoolismo é um problema de saúde pública de dimensão ainda não bem conhecida no Brasil, que traz como consequiência doenças orgânicas graves, o que torna necessária uma preocupação efetiva com o seu conhecimento, tratamento e prevenção.

Neste sentido, como em outros países, foram desenvolvidos estudos que objetivaram contribuir no esclarecimento da sua amplitude e características $4,8,8,8,10,11$.

O brasileiro é um grande consumidor de bebidas alcoólicas. Segundo dados de
1981, o Brasil é o $13 .^{\circ}$ maior consumidor de bebidas do mundo e o primeiro em bebidas destiladas ${ }^{1}$. No ano de 1983 a população brasileira adulta, 73 milhões de pessoas, ingeriu mais de 3 bilhões de litros de bebidas alcoólicas ${ }^{3}$.

Programas de prevenção dos efeitos do alcoolismo na população têm sido desenvolvidos pela Organização Mundial de Saúde ${ }^{13}$, de prática difícil seja pela facilidade na obtenção da bebida, baixo custo, pelo fato de fornecer calorias ou pelo seu caráter simbólico ${ }^{8}$.

\footnotetext{
- Do Departamento de Clínica Médica da Faculdade de Medicina de Ribeirão Preto da Universidade de São Paulo. Campus da USP de Ribeirăo Preto - 14:100 - Ribeirão Preto, SP - Brasil.
} 
DANTAS, R.O. Tempo de alcoolismo no desenvolvimento de doenças orgânicas em mulheres tratadas no Hospital das Clínicas de Ribeirão Preto, SP, Brasil. Rev. Saúde públ,. S. Paulo, 19:304-10, 1985.

As suas influências negativas na vida do país fez com que fossem criados grupos de trabalho para o estudo do problema, o qual preocupa até mesmo as indústrias de bebidas ${ }^{3}$.

E conhecido que entre as mulheres as consequiências do alcoolismo aparecem com miaior rapidez e gravidade ${ }^{2,7,15,16}$.

Como no Brasil há poucos estudos das consequiências do alcoolismo entre mulheres, estudamos o tempo desse hábito no aparecimento de doenças orgânicas nas mulheres tratadas no Hospital das Clínicas de Ribeirão Preto, a semelhança do que fizemos para homens da mesma população ${ }^{4,5}$.

\section{MATERIAL E MÉTODOS}

Estudamos 95 mulheres portadoras de doenças orgânicas consequientes ao alcoolismo, tratadas entre 1978 e 1982 no Hospital das Clínicas de Ribeirão Preto.

As pacientes tinham diagnóstico de pelagra, desnutrição, neurite periférica, cirrose hepática, hepatite alcoólica ou pancreatite crônica, tratadas nas enfermarias de Clínica Médica, não sendo incluídas aquelas com somente sintomas psiquiátricos.

$\mathrm{Na}$ época do início do tratamento elas ingeriam bebidas alcoólicas e podiam ser classificadas como bebedoras-problemas na classificação de Naveillan ${ }^{14}$. A bebida que ingeriam era a mais comum na região, a aguardente (pinga), e apenas ocasionalmente outro tipo de bebida.

O diagnóstico de pancreatite crônica foi feito pela presença de crises de dores abdominais com níveis elevados de amilase e lipase no sangue, podendo ainda estar presente calcificações pancreáticas, vistas no exame radiológico do abdômen, e sinais e sintomas de insuficiência pancreática. Foram afastadas outras etiologias, que não o alcoolismo, para justificar a pancreatite.

Cirrose hepática foi diagnosticada pela presença de sinais de hipertensão portal (esplenomegalia, ascite, circulação colateral no abdômen e varizes de esôfago), hipoalbuminemia, insuficiência hepática e, em alguns casos, por biópsia hepática.

As outras pacientes (alcoolismo crônico com complicações médicas) tinham pelagra, desnutrição, neurite periférica ou hepatite alcoólica, sem sinais ou sintomas de pancreatite ou cirrose.

Foi realizada em todas observação clínica e exame físico. A evolução da doença e exames complementares confirmaram o diagnóstico.

Alguns dias após a internação indagamos sobre a idade em que iniciaram a ingestão de bebidas alcoólicas (frequiência de ingestão média de mais de $100 \mathrm{ml}$ de etanol por dia, pelo menos três dias por semana), a que chamamos início do beber excessivo (BE), a idade em que iniciaram os sinais e sintomas que motivaram a procura de tratamento médico (ID), e outros dados pessoais e familiares.

Para comparar os resultados entre as doenças estudadas utilizamos o teste $\mathrm{U}$ de Mann-Whitney.

\section{RESULTADOS}

O diagnóstico de cirrose hepática foi feito em 32 pacientes, pancreatite crônica em 13 e outras doenças em 50 .

A distribuição das idades em que iniciaram a ingestão frequiente de bebidas alcoólicas, do início dos sinais e sintomas das doenças orgânicas consequientes ao alcoolismo e o tempo de alcoolismo, está apresentada nas Tabelas 1 e 2. A média, desvio padrão e mediana destes parâmetros estão apresentados na Tabela 3.

Não houve diferenças entre as idades 
DANTAS, R.O. Tempo de alcoolismo ns desenvolvimento de doenças orgânicas em mulheres tratadas no Hospital das Clínicas de Ribeirão Preto, SP, Brasil. Rev. Saúde públ,. S. Paulo, 19:304-10, 1985 .

para os três grupos de doenças, bem como para o tempo de alcoolismo $(\mathbf{P}>$ $0,05)$.

O estado civil, a alfabetização e a existência de história familiar de alcoolismo estão relacionados na Tabela 4.

Nesta tabela percebe-se que $64 \%$ das mulheres referiam que tinham familiares que ingeriam bebidas alcoólicas frequientemente. Este familiar foi, na maioria das vezes, o marido ou companheiro, e menos frequientemente o pai.

Foi destacante a não referência por nenhuma delas da presença de outras mulheres na família com problemas relacionados com o alcoolismo.

A alfabetização referida na Tabela 4 correspondeu ao fato de saber ler e escrever, independente do grau de escolaridade atingido.

\section{TABELA 1}

Distribuição (anos) das idades do início do beber excessivo (BE) e do aparecimento dos sinais e sintomas das doenças provocadas pelo alcoolismo (ID), de mulheres tratadas no Hospital das Clínicas de Ribeirão Preto, SP.

\begin{tabular}{|c|c|c|c|c|c|c|c|c|c|c|c|c|}
\hline \multirow{3}{*}{$\begin{array}{l}\text { Idade } \\
\text { (anos) }\end{array}$} & \multicolumn{4}{|c|}{$\begin{array}{c}\text { Alcoolismo crônico } \\
\text { com complicações } \\
\text { médicas }\end{array}$} & \multicolumn{4}{|c|}{$\begin{array}{c}\text { Pancreatite crônica } \\
\text { alcoólica }\end{array}$} & \multicolumn{4}{|c|}{$\begin{array}{c}\text { Cirrose hepática } \\
\text { alcoólica }\end{array}$} \\
\hline & \multicolumn{2}{|c|}{$\mathrm{BE}$} & \multicolumn{2}{|c|}{ ID } & \multicolumn{2}{|c|}{$\mathrm{BE}$} & \multicolumn{2}{|c|}{ ID } & \multicolumn{2}{|c|}{$\mathrm{BE}$} & \multicolumn{2}{|c|}{ ID } \\
\hline & N.o & $\%$ & N.o & $\%$ & N. ${ }^{\circ}$ & $\%$ & N.o & $\%$ & N. ${ }^{\circ}$ & $\%$ & N. ${ }^{\circ}$ & $\%$ \\
\hline 0. 9 & 4 & 8 & 0 & 0 & 0 & 0 & 0 & 0 & 2 & 6 & 0 & 0 \\
\hline $10-19$ & 20 & 40 & 2 & 4 & 5 & 38 & 0 & 0 & 10 & 31 & 0 & 0 \\
\hline $20-29$ & 19 & 38 & 14 & 28 & 8 & 62 & 3 & 23 & 13 & 41 & 6 & 19 \\
\hline $30-39$ & 4 & 8 & 26 & 52 & 0 & 0. & 7 & 54 & 5 & 16 & 16 & 50 \\
\hline $40-49$ & 3 & 6 & 3 & 6 & 0 & 0 & 2 & 15 & 2 & 6 & 8 & 25 \\
\hline $50-60$ & 0 & 0 & 5 & 10 & 0 & 0 & 1 & 8 & 0 & 0 & 2 & 6 \\
\hline
\end{tabular}

TABELA 2

Tempo de beber excessivo até o aparecimento da doença em mulheres tratadas no Hospital das Clínicas de Ribeirão Preto, SP.

\begin{tabular}{rrrrrrr}
\hline \multirow{2}{*}{$\begin{array}{c}\text { Tempo } \\
\text { (anos) }\end{array}$} & $\begin{array}{c}\text { Alcoolismo crônico } \\
\text { com complicações } \\
\text { médicas }\end{array}$ & \multicolumn{2}{c}{$\begin{array}{c}\text { Pancreatite crônica } \\
\text { alcoólica }\end{array}$} & \multicolumn{2}{c}{$\begin{array}{c}\text { Cirrose hepática } \\
\text { alcoólica }\end{array}$} \\
\cline { 2 - 7 } & N..$^{\circ}$ & $\%$ & N. ${ }^{\circ}$ & $\%$ & N. ${ }^{\circ}$ & $\%$ \\
\hline $0-9$ & 18 & 36 & 2 & 15 & 8 & 25 \\
$10-19$ & 19 & 38 & 8 & 62 & 13 & 41 \\
$20-29$ & 11 & 22 & 2 & 15 & 10 & 31 \\
$30-39$ & 1 & 2 & 1 & 8 & 1 & 3 \\
$40-50$ & 1 & 2 & 0 & 0 & 0 & 0 \\
\hline Total & 50 & 100 & 13 & 100 & 32 & 100 \\
\hline
\end{tabular}


DANTAS, R.O. Tempo de alcoolismo no desenvolvimento de doenças orgânicas em mulheres tratadas no Hospital das Clínicas de Ribeirão Preto, SP, Brasil. Rev. Saúde públ,. S. Paulo, 19:304-10, 1985 .

\section{TABELA 3}

Média ( $\overline{\mathbf{X}}$ ), desvio padrão (S) e mediana (M), em anos, das idades do início do beber excessivo (BE), do aparecimento dos sinais e sintomas das doenças provocadas pelo alcoolismo (ID) e do tempo de beber excessivo (T) até o aparecimento da doença em mulheres tratadas no Hospital das Clínicas de Ribeirão Preto, SP.

\begin{tabular}{|c|c|c|c|c|c|c|c|c|c|}
\hline \multirow[t]{2}{*}{ Valores } & \multicolumn{3}{|c|}{$\begin{array}{c}\text { Alcoolismo crônico } \\
\text { com complicações } \\
\text { médicas }\end{array}$} & \multicolumn{3}{|c|}{$\begin{array}{c}\text { Pancreatite crônica } \\
\text { alcoólica }\end{array}$} & \multicolumn{3}{|c|}{$\begin{array}{l}\text { Cirrose hepática } \\
\text { alcoólica }\end{array}$} \\
\hline & $\mathrm{BE}$ & ID & $\mathbf{T}$ & $\mathrm{BE}$ & ID & $\mathrm{T}$ & $\mathrm{BE}$ & ID & $T$ \\
\hline $\bar{x}$ & 21,48 & 33,90 & 13,24 & 19,38 & 35,30 & 15,92 & 22,03 & 36,53 & 14,62 \\
\hline$s$ & 9,00 & 11,27 & 9,58 & 2,98 & 7,72 & 7,15 & 9,25 & 8,39 & 8,70 \\
\hline $\mathbf{M}$ & 20,00 & 33,00 & 12,50 & 20,00 & 33,00 & 15,00 & 21,00 & 36,00 & 12,50 \\
\hline
\end{tabular}

TABELA 4

Estado civil, alfabetização e história familiar de alcoolismo das mulheres tratadas no Hospital das Clínicas de Ribeirão Preto, SP.

\begin{tabular}{|c|c|c|c|c|c|c|}
\hline \multirow[b]{2}{*}{ Doenças } & \multicolumn{2}{|c|}{ Estado Civil } & \multicolumn{2}{|c|}{ Alfabetização } & \multicolumn{2}{|c|}{ História Familiar } \\
\hline & Casada & Solteira & Sim & Não & Presente & Ausente \\
\hline Cirrose hepática & 22 & 10 & 15 & 17 & 22 & 10 \\
\hline Pancreatite crônica & 6 & 7 & 7 & 6 & 7 & 6 \\
\hline Outras doenças & 22 & 28 & 18 & 32 & 32 & 18 \\
\hline Total & 50 & 45 & 40 & 55 & 61 & 34 \\
\hline
\end{tabular}

\section{DISCUSSÃO E CONCLUSÕES}

Comparando os resultados aqui apresentados com aqueles obtidos para homens da mesma população ${ }^{4,5}$, vemos que, em média, há tendência a que as mulheres demorem menos tempo para as manifestações das doenças orgânicas consequientes ao alcoolismo. Entre os homens este tempo foi de 18,32 anos nos pacientes com alcoolismo crônico com complicações médicas, 15,85 nos pacientes com pancreatite crônica e 21,10 nos pacientes com cirrose hepática ${ }^{5}$, enquanto que nas mulheres ele foi de 13,24 ,
15,92 e 14,62 anos, respectivamente. Como indicam estes resultados a tendência a tempo menor nas mulheres não acontece nos pacientes com pancreatite crônica, talvez pela necessidade de estudo de um maior número de mulheres com este diagnóstico, ou que nas mulheres o aparecimento da pancreatite não seja mais rápido que nos homens, ao contrário do que acontece principalmente nas doenças hepáticas.

$\mathrm{Na}$ análise da Tabela 1 vemos que 6 mulheres $(6 \%)$ referiram estar ingerindo bebidas alcoólicas em grande quantidade 
DANTAS, R.O. Tempo de alcoolismo no desenvolvimento de doenças orgânicas em mulheres tratadas no Hospital das Clínicas de Ribeirão Preto, SP, Brasil. Rev. Saúde públ,. S. Paulo, 19:304-10, 1985.

antes dos 10 anos de idade, proporção semelhante aos $5 \%$ que encontramos entre os homens ${ }^{5}$. Provavelmente estes pacientes ingeriam bebidas alcoólicas com esta idade, porém em quantidades inferiores a que referiram na entrevista. A avaliação da quantidade ingerida apresenta dificuldades porque ela não é constante com o decorrer do tempo. Estabelecemos uma quantidade mínima que pudesse servir como referência do início da ingestão excessiva da bebida, todavia na época do tratamento no hospital a grande maioria referia estar bebendo quantidades bem superiores.

No caso destas 6 pacientes houve internações no hospital, causadas por hepatopatia alcoólica, antes dos 30 anos de idade.

Mais de $65 \%$ das mulheres tiveram tempo de alcoolismo inferior a 20 anos e antes dos 40 anos estavam em tratamento das doenças provocadas pelo hábito. Entre os homens menos de $60 \%$ tiveram tempo de alcoolismo inferior a 20 anos e início das manifestações das doenças antes dos 40 anos ${ }^{5}$.

Entre pacientes internados em hospitais gerais do Brasil a frequiência $e$ volume de ingestão de bebidas alcoólicas são maiores entre os homens ${ }^{9,10,11}$, mas é sabido que a gravidade da hepatite alcoólica é maior entre as mulheres, mesmo que ela não consuma quantidades muito elevadas?.

Estudo feito em hospital geral encontrou que mulheres com indicadores de alcoolismo tinham idade inferior aquelas sem indicadores e que nos homens a idade foi praticamente a mesma entre aqueles com ou sem indicadores de alcoolismo ${ }^{10}$.

No mesmo sentido, trabalho em que foi estudada a mortalidade por cirrose hepática refere a idade da morte, pela doença, de 56,3 anos nos homens e 48,6 anos nas mulheres ${ }^{16}$.

Comparando o efeito do álcool em relação ao sexo, Ashley e col. ${ }^{2}$ encontraram que a idade em que iniciaram a ingestão de bebidas alcoólicas foi praticamente a mesma entre homens e mulheres, mas os homens ingeriam quantidades maiores com menos idade. $O$ tempo entre o início da ingestão até a internação foi de $20,2 \pm 9,3$ anos nos homens e 14,1 $\pm 8,5$ anos nas mulheres, com tempo menor para elas terem certas complicações como hemorragia digestiva, desnutrição, esteatose hepática, anemia e hipertensão arterial.

Se nas mulheres o alcoolismo causa maior rapidez no aparecimento de doen. ças, é importante que ela interrompa a ingestão para melhorar o prognóstico. E conhecido que a interrupção do hábito retarda a evolução da doença hepática, e que quando isto não ocorre o tempo de sobrevida diminui acentuadamente, principalmente em mulheres ${ }^{7}$. A interrupção do alcoolismo tem particular importância durante a gravidez porque a sua manutenção pode provocar o nascimento de crianças com lesões congênitas graves ${ }^{8,12}$. Há no Canadá um programa de esclarecimento das mulheres grávidas dos riscos do alcoolismo durante a gestação.

$\mathrm{O}$ encontro de referência a alcoolismo em outras pessoas da família em $64 \%$ dos casos que estudamos pode justificar a má evolução que frequientemente acontece nestas pacientes, porque o convívio deve dificultar o abandono do hábito.

As justificativas para a progressão da doença hepática ser mais rápida nas mulheres está em possível diferença no volume ingerido, padrão de ingestão, diferença no metabolismo do etanol ou pela presença de maior quantidade de imunoglobulinas no soro das mulheres? 
DANTAS, R.O. Tempo de alcoolismo no desenvolvimento de doenças orgânicas em mulheres tratadas no Hospital das Clínicas de Ribeirão Preto, SP, Brasil. Rev. Saúde públ,. S. Paulo, 19:304-10, 1985 .

DANTAS, R.O. [Effect of time of alcoholism on the development of organic diseases in women treated at the University Hospital of Ribeirão Preto, S. Paulo, Brazil]. Rev Saúde públ., S. Paulo, $19: 304-10,1985$.

ABSTRACT: To determine the period of frequent alcoholic drink intake (mean intake of $100 \mathrm{ml}$ of ethanol per day on at least three days a week) up to the onset of signs and symptoms of organic diseases caused by the habit, 95 women treated at the University Hospital of the Faculty of Medicine of Ribeirão Preto for organic diseases associated with alcoholism between 1978 and 1982, we studied. Clinical and laboratory diagnosis of cirrhosis of the liver was made in 32 patients, of chronic pancreatitis in 13, and of other diseases (pellagra, malnutrition, peripheral neuritis, and alcoholic hepatitis) in 50. Patients showing only psychiatric symptoms were excluded from the study. Patient history was obtained after a few days of treatment. On average, the age when signs and symptoms of the diseases that motivated them to seek hospital treatment began to appear was $35.30 \pm 7.72$ years for chronic pancreatitis patients, $36.53 \pm 8.39$ for patients with cirrhosis of the liver, and $33.90 \pm 11.27$ for the patients with other diseases. Time of alcohol intake was $15.92 \pm 7.15$ years for chronic pancreatitis patients, $14.62 \pm 8.70$ years for patients with cirrhosis of the liver, and 13.24 \pm 9.58 years for patients with other diseases. A family history ol afcoholism was present in $64.2 \%$ of cases and generally involved the husband. None of the patients reported other women with problems of alcoholism in the family. The period of alcoholism before the onset of cirrhosis of the liver among the women studied (14.62 years) was shorter than that encountered for men from the same population $(21.10$ years), studied in previous investigations.

UNITERMS: Alcoholism. Women. Alcohol drinking. Liver cirthosis, alcoholic. Pancreatitis.

\section{REFERENCIAS BIBLIOGRÁFICAS}

1. A ameaça engarrafada. Veja, 21 out., 1981. p. 88-96.

2. ASHLEY, M.J.; OLIN, J.S.; RICHE, H. W.; KORNACZEWSKI, A.; SCHMIDT, W. \& RANKIN, J.G. Morbidity in alcoholics. Arch. intern. Med., 137:883-7, 1977.

3. COCIUFFO, S. Alcoolismo leva o país ao estado de alerta. Rev. bras. Clin. lerap., 13:420-8, 1984.

4. DANTAS, R.O. Alcoolismo entre trabalhadores rurais e urbanos. Cienc. Cult. 31:774-6, 1979.

5. DANTAS, R.O. Alcoolismo em trabalhadores da zona urbana e rural. Uma experiência em Brasil. Bol. Ofic, sanit. panamer, 94:76-82, 1983.

6. HANSON, J.W.; JONES, K.L. \& SMITH, D.W. Fetal alcohol syndrome. J. Amer. med. Ass., 235-1458-60, 1976.

7. KRASNER, N.; DAVIS, M.; PORTMANN, B. \& WILLIAMS, R. Changing pattern of alcoholic liver disease in Great Britain: relation to sex and signs of autoimmunity. Brit. med. J., 1:1497. $500,1977$.

8. MASUR, J. Conjecturas sobre o uso milenar de bebidas alcoólicas. Cienc. Cult., 30:531-4, 1978.

9. MASUR, J.; TUFIK, S.; RIBEIRO, A.B.; SARAGOÇA, M.A.S. \& LARANJEIRA, R.R. Consumo de álcool em pacientes de hospital geral: um problema negligenciado? Rev. Ass, med. bras., 25:3026. 1979.

10. MASUR, J.; CUNHA, J.M.; ZWICKER, A.P.; LARANJEIRA, R.R.; KNOBEL, E.; SUSTOVICH, D.R. \& LOPES, A.C. Prevalência de pacientes com indicadores de alcoolismo internados em uma enfermaria de clínica geral. Relevância da forma de detecção. Acta psiquiat. psical. Amer. Lat., 26:125-30, 1980.

11. MOREIRA, L.F.S.; CAPRIGLIONE, M. J. \& MASUR, J. Consumo de álcool em pacientes ambulatoriais de hospital geral na capital e interior do Estado do Rio Grande do Norte. Rev. Ass. bras. Psiq., 2:183-9, 1980. 
DANTAS, R.O. Tempo de alcoolismo no desenvolvimento de doenças orgânicas em mulheres tratadas no Hospital das Clínicas de Ribeirão Preto, SP, Brasil. Rev. Saúde públ,. S. Paulo, 19:304-10, 1985.

12. MORRISON, A.B. \& MAYKUT, M.O. Potential adverse effects of maternal alcohol ingestion on the developing fetus and their sequelae in the infant and child. Canad. med. Ass. J., 120:826-8, 1979.

13. MOSER, J. Prevention of alcohol: related problems. Toronto, World Health Organization, 1980.

14. NAVEILLAN, F.P. Sobre el concepto de alcoholismo. Bol. Ofic. sanit. panamer., 91:340-8, 1981.
15. NOLAN, J.P.Alcohol as a factor in the illness of university service patients. Amer. J. med. Sci., 249:135-42, 1965.

16. SPAIN, D.M. Portal cirrhosis of the liver. Amer. J. clin. Path., 15:215-8, 1945.

Recebido para publicação em 04/01/1985

Reapresentado em 30/05/1985

Aprovado para publicação em 03/06/1985 